\title{
Glucose transporter 3 performs a critical role in mTOR-mediated oncogenic glycolysis and tumorigenesis
}

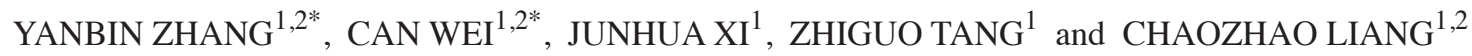 \\ ${ }^{1}$ Department of Urology, The First Affiliated Hospital of Anhui Medical University, Hefei, Anhui 230032; \\ ${ }^{2}$ Department of Urology, Hefei Hospital Affiliated to Anhui Medical University and The Second People’s Hospital of Hefei, \\ Hefei, Anhui 230011, P.R. China
}

Received July 13, 2014; Accepted March 2, 2015

DOI: $10.3892 / \mathrm{ol} .2015 .3075$

\begin{abstract}
The present study aimed to examine the relationship between mammalian target of rapamycin (mTOR) and glucose transporter 3 (Glut3) in the process of mTOR-mediated oncogenic glycolysis and tumorigenesis. Western blot analysis and quantitative polymerase chain reaction were used to compare the expression of Glut 3 in mouse embryonic fibroblasts (MEFs) null for tuberous sclerosis complex $2\left(\mathrm{Tsc}^{-{ }^{--}}\right)$and control $T s c 2^{+/+}$MEFs. In addition, the glycolytic rate was tested following siRNA-mediated knockdown of Glut3 in $T s c 2^{-1}$ cells. To determine whether Glut 3 depletion affects the ability of cells to form tumors in vivo. Tsc $2^{-/-}$MEFs infected shGlut3 and shControl were injected into nude mice subcutaneously. The present study demonstrated that the expression of Glut3 is controlled by mTOR in $T s c 2^{-/}$cells and that downregulation of Glut 3 reduced the glycolytic rate in $T s c 2^{-1-}$ cells. cells. Further studies in nude mice demonstrated that reduced Glut3 expression levels reduced the tumorigenetic effect in cells with hyperactive mTOR complex 1 (mTORC1). The present study indicates for the first time that Glut3 is a downstream target of mTORC1 and that Glut 3 is critical in mTORC1-associated tumorigenesis. Therefore, Glut 3 is a potential target for the treatment of diseases associated with dysregulated mTORC1 signaling.

Introduction
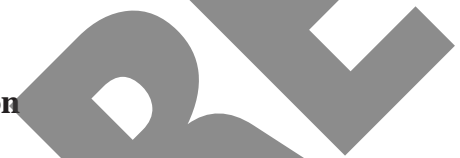

The process of glycolysis provides the majority of the energy required by cancer cells, however it is an inefficient process for energy production. To offset this disadvantage, the glycolytic rate in tumor cells is $100 \mathrm{x}$ higher than that in normal cells,

Correspondence to: Dr Chaozhao Liang, Department of Urology, The First Affiliated Hospital of Anhui Medical University, 218 Jixi Road, Hefei, Anhui 230032, P.R. China

E-mail: docliangchaozhao@163.com

"Contributed equally
\end{abstract}

Key words: mTOR, Glut3, glycolysis, tumorigenesis and glucose uptake into tumor cells is also accelerated (1). A family of glucose transporters (Gluts) facilitates glucose movement across the plasma membranes in a tissue-specific manner, and 14 different Glut isoforms have been characterized at present (2). Glut3 is an effective glucose transporter, preyious studies have demonstrated that Glut3 is upregulated in certain types of malignancy and that it is associated with poor prognosis $(3,4)$. Downregulation of Glut3 reduces the glucose consumption of the cell and therefore cell growth rate (5). However, the underlying mechanisms that lead to the upregulation of Glut 3 in tumor cells remain unclear.

Mammalian target of rapamycin (mTOR) serves a critical function in the carcinogenic phosphoinositide 3-kinase $(\mathrm{P} 3 \mathrm{~K}) /$ protein kinase B (AKT)/mTOR signaling pathway (6). In mammalian cells, mTOR partners with different proteins to form two functionally distinct complexes, mTOR complex 1 (mTORC1) and mTORC2. mTORC1 is rapamycin-sensitive and activated by the PI3K/AKT pathway, whereas mTORC2 is not sensitive to rapamycin. Previous studies have demonstrated the critical role of mTORC1 in glycolysis during tumorigenesis, however, as with Glut3, the underlying mechanism remains unclear $(7,8)$.

The aim of the present study was to assess the association between Glut 3 and mTORC1, including observation of glycolytic rate in mouse embryonic fibroblasts (MEFs) and tumor growth in nude mice.

\section{Materials and methods}

Reagents, plasmids and antibodies. Rapamycin, Lipofectamine 2000 and 4-12\% Bis-Tris Nu-PAGE gels were purchased from Invitrogen Life Technologies (Carlsbad, CA, USA). The pLL3.7-GFP lentiviral vector was a gift from Professor Xiaojun Zha (Anhui Medical University, Hefei, China). TSC2 (catalog no. SKU3611S; polyclonal, rabbit anti-human; dilution 1:250), $\beta$-actin (catalog no. SKU12262S; monoclonal, mouse anti-human; dilution 1:300), S6 (catalog no.SKU6989S; monoclonal, mouse anti-human; dilution 1:400), p-S6 (Ser235/236; catalog no. SKU5202S; monoclonal, mouse anti-human; dilution 1:300), mTOR (catalog no. SKU2972S; polyclonal, rabbit anti-human; dilution 1:150), Raptor (catalog no. SKU2280P; monoclonal, rabbit anti-human; dilution 1:300) and Rictor (catalog no. SKU2114P; monoclonal, rabbit 
anti-human; dilution 1:400) antibodies were purchased from Cell Signaling Technology, Inc. (Danvers, MA, USA).

Cell cultures. All mouse embryonic fibroblasts (MEFs) used in the study were purchased from Clontech Laboratories, Inc. (Mountainview, CA, USA). HEK293T cells were obtained from the American Type Culture Collection (Manassas, VA, USA). All cells were cultured in Dulbecco's modified Eagle's medium (DMEM; Maixin Bio, Fujian, China) with 10\% fetal bovine serum and $1 \%$ penicillin/streptomycin (purchased from Maixin Bio) in $5 \% \mathrm{CO}_{2}$ at $37^{\circ} \mathrm{C}$.

Plasmid construction, lentiviral transfection and transduction. pLL3.7-GFP plasmids were digested with $\mathrm{XhoI}$ and $\mathrm{HpaI}$ restriction enzymes (Takara Biotechnology Co., Ltd., Dalian, China) followed by insertion of annealed oligonucleotides using T7 DNA Ligase (MCLAB, San Francisco, CA, USA). The sequences of the oligonucleotides were as follows: Glut $3^{1}$, F 5'-TCAACAGGAATCTTCAAGGATTCAAGAGATCCT TGAAGATTCCTGTTGTTTTTTC-3', and R 5'-TCGAGA AAAAACAACAGGAATCTTCAAGGATCTCTTGAATCC TTGAAGATTCCTGTTGA-3'; Glut3², F 5'-TGGAGGAAG ACCAAGCTACATTCAAGAGATGTAGCTTGGTCTTCC TCCTTTTTTC-3' and R 5'-TCGAGAAAAAAGGAGGAA GACCAAGCTACATCTCTTGAATGTAGCTTGGTCTTC CTCCA-3'; control, F 5'-TGTACTGCAGCCACATTCTCT TCAAGAGAGAGAATGTGGCTGCAGTACTTTTTTC-3' and R 5'-TCGAGAAAAAAGTACTGCAGCCACATTCTC TCTCTTGAAGAGAATGTGGCTGCAGTACA-3'. Th HEK293T cells were transfected with the pLL3.7-GFP vector containing either of these sequences together with the packaging vectors (pVSVG, pREV and pMDL, purchased from Shanghai GenePharma Co., Ltd.; Shanghai, China). Culture supernatants were collected after 48- and 72-h transfection and then used to infect $T s{ }^{2-\digamma}$ MEFs.

Reverse transcription-polymerase chain reaction ( $R T-P C R)$ and RT-quantitative (q)PCR. After extracting from the cells with Trizol solution (Invitrogen Life Technologies) and purifying using the RNAeasy Maxi Kit (Qiagen, Hilden, Germany) according to the manufacturer's instructions, $1 \mu \mathrm{g}$ mRNA for each sample was used for reverse transcription with Omniscript RT kit (Qiagen). RT-PCR and qPCR were performed according to the manufacturer's protocol. RT-PCR analysis of Glut 3 with $\beta$-actin as a control was conducted using the following primers: Glut3, F 5'-ATGGGGACAACG AAGGTGACCCATCTCTG-3' and R 5'-TGAGCTACCAGA ATCCAACAACG-3'; $\beta$-actin, F 5'-ATGGATGACGATATC GCTGCGC-3' and R 5'-GCAGCACAGGGTGCTCCTCA-3'. For RT-qPCR, the primer sequences were as follows: Glut3, F 5'-TGGTAGCTCAGATCTTTGGTTTGG-3' and R 5'-GAT CTCTGTAGCTTGGTCTTCCTC-3'; $\beta$-actin, F 5'-AGAGGG AAATCGTGCGTGAC-3' and R 5'-CAATAGTGATGACCT GGCCGT-3'. qRT-PCR was performed and analyzed using the ABI PRISM 7000 Fluorescent Quantitative PCR System (Applied Biosystems Life Technologies, Foster City, CA, USA). A total reaction volume of $20 \mu \mathrm{l}$ was used, consisting of $1 \mu \mathrm{l}$ of cDNA template synthesized previously, $10 \mu \mathrm{l}$ SYBR Green master mix (Invitrogen Life Technologies), $1 \mu 1$ of each primer ( $\mathrm{F}$ and $\mathrm{R}$ primer) and RNase-free water. Cycling parameters were set as $95^{\circ} \mathrm{C}$ for $2 \mathrm{~min}$, followed by 40 cycles of $95^{\circ} \mathrm{C}(15 \mathrm{sec}), 55^{\circ} \mathrm{C}(30 \mathrm{sec})$, and $72^{\circ} \mathrm{C}(40 \mathrm{sec})$.

Western blot analysis. Western blot analysis of protein expression was performed using a NE-PER Nuclear and Cytoplasmic Extraction Reagent kit (Pierce Biotechnology, Inc., Rockford, IL, USA) according to the manufacturer's instructions. The cells were lysed in lysis buffer (2\% sodium dodecyl sulfate, $10 \%$ glycerol, $10 \mathrm{mM}$ Tris, $\mathrm{pH} 6.8,100 \mathrm{mM}$ dithiothreito; purchased from Maixin Bio), boiled for $10 \mathrm{~min}$, and subsequently subjected to immunoblotting with the following steps: Proteins (15 $\mu \mathrm{l})$ were loaded onto a 10\% SDS gel, and were separated at $160 \mathrm{~V}$ for $70 \mathrm{~min}$. Proteins were then transferred onto a PVDF membrane. The membranes were blocked with 5\% non-fat milk in Tris-buffered saline and Tween 20 (TTBS) solution for $1 \mathrm{~h}$ at RT with agitation, and subsequently rinsed with TTBS solution twice. Membranes were incubated with primary antibodies overnight at room temperature, and subsequently incubated with $2 \mu \mathrm{l}$ anti-mouse $\lg \mathrm{G}$ secondary antibody for $1 \mathrm{~h}$ at room temperature with agitation. Membranes were developed with enhanced chemiluminescence reagents, and exposed to high sensitivity X-ray films in the dark. Analyses of the western blot results were subsequently performed using the Quantity One software, version 4.62 (Bio-Rad Laboratories, Inc., Hercules, CA, USA). Protein sample preparation, electrophoresis, transfer, blocking, primary antibody incubation, secondary antibody incubation and then detection of proteins. Analyses of the western blot results were subsequently performed using the Quantity One software (Bio-Rad Laboratories, Inc., Hercules, CA, USA).

$R N A$ interference. $T s c 2^{--} \mathrm{MEFs}$ cells were seeded at a density of $5 \times 10^{5} / \mathrm{ml}$ in DMEM in 12-well plates and were transfected with siRNAs using Lipofectamine 2000 (Invitrogen Life Technologies) according to the manufacturer's instructions. All the siRNA oligonucleotides were synthesized by Shanghai GenePharma Co., Ltd. (Shanghai, China). siRNA targeted sequences were as follows: mTOR, 5'-GAACTCGCTGAT CCAGATG-3'; Raptor, 5'-GGACAACGGTCACAAGTAC-3'; Rictor, 5'-GCCCTACAGCCTTCATTTA-3'; p65, 5'-GGACCT ATGAGACCTTCAA-3'; and negative control, 5'-TTCTCC GAACGTGTCACGT-3'.

Measurements of glucose consumption and lactate production. The $T s{ }^{-/}$cells expressing shControl and shGlut 3 were seeded at a density of $1 \times 10^{6} / \mathrm{ml}$. in DMEM in culture dishes and cultured for $8 \mathrm{~h}$. The culture medium was replaced with fresh medium and cells were incubated for an additional $16 \mathrm{~h}$. Subsequently, the culture medium was collected and used to determine the glucose and lactate concentration using a Glucose Assay kit and a Lactate Assay kit (Eton Bioscience, Inc., San Diego, CA, USA).

Induction of subcutaneous tumors in nude mice. Immunodeficient nude male mice ( $\mathrm{n}=22, \mathrm{BALB} / \mathrm{c}, 6$-week-old; weight, $18-20 \mathrm{~g}$ ) were maintained and used in strict accordance with the guidelines of the Animal Center of Anhui Medical University (Anhui, China). Six male mice were used in each cohort. Tumor growth and mouse survival were assessed over 1-2-month periods following subcutaneous inoculation of 
A
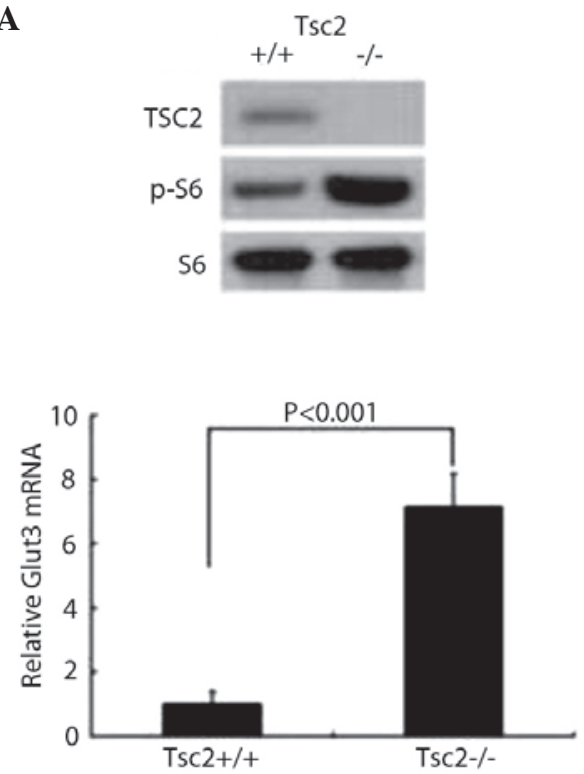
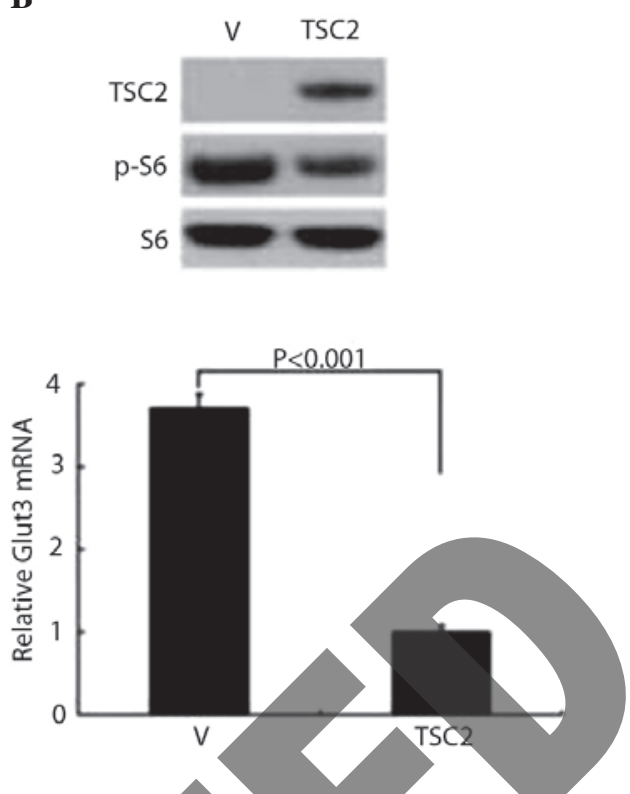

Figure 1.TSC2/mTOR promote the expression of Glut3.(A) Tsc2 $2^{+/+}$and $T s c 2^{--}$MEFs; (B) Tsc2--MEFs were infected with pLXIN-hygro (V) or pLXIN-TSC2 (TSC2) retroviruses. Upper panels, cell lysates were subjected to immunoblotting with the indicated antibodies; lower panels, reverse transcription-quantitative polymerase chain reaction was performed to detect the expression of Glut3. Error bars indicate the mean \pm standard deviation of triplicate samples. MEFs, mouse embryonic fibroblasts; mTOR, mammalian target of rapamycin; Glut3, glucose transporter 3; Tsc2, tuberous sclerosis complex 2.

A
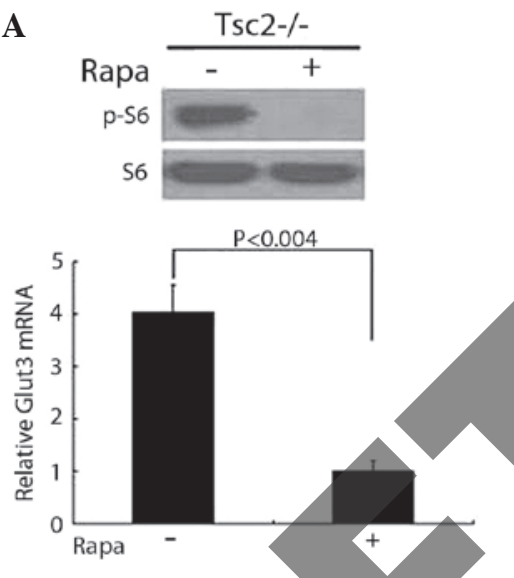

B

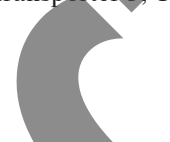

C Tsc2- $\%$

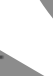

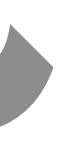

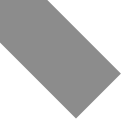

Figure 2. mTORC1 is responsible for the promotion of Glut3 expression. (A) Tsc2 $2^{-}$MEFs were treated with or without $10 \mathrm{nM}$ rapamycin (Rapa) for $24 \mathrm{~h}$. (B) $T s c 2^{--}$MEFs were transfected with mTOR, Raptor or Rictor siRNAs for $48 \mathrm{~h}$. The proteins were detected by immunoblotting with the indicated antibodies. (C) The mRNA level of Glut 3 was analyzed by reverse transcription-quantitative polymerase chain reaction. Error bars indicate the mean \pm standard deviation of triplicate samples. mTORC1, mammalian target of rapamycin complex 1; Glut3, glucose transporter 3; Tsc2, tuberous sclerosis complex 2; MEFs, mouse embryonic fibroblasts; siNC, normal control siRNA.

$1 \times 10^{6}$ cells $\left(T s c 2^{-/-}\right.$MEFs infected with shGlut31 or shControl) in $0.2 \mathrm{ml}$ DMEM into the right posterior back region. Following inoculation, the mice were examined at least twice weekly for tumor development by palpation. Survival was determined by the requirement for euthanasia due to a tumor size $>1,000 \mathrm{~mm}^{3}$, ulceration of the tumor or weight loss of $>10 \%$. All animal protocols were approved by the Center for Animal Resources and Comparative Medicine of Anhui Medical University, and were compliant with the university guidelines on the care of experimental animals.

Statistical analysis. The data were analyzed using a 2-tailed paired Student's t-test. The Kaplan-Meier log-rank test was used for analysis of tumor development and survival data. In all tests, $\mathrm{P}<0.05$ was considered to indicate a statistically significant difference and all statistical analyses were performed with the SPSS software package, version 17.0 (SPSS, Inc., Chicago, IL, USA).

\section{Results}

TSC2/mTOR promote the expression of Glut3. Tuberous sclerosis complex is caused by mutations in TSC1 or TSC 2 genes. In the PI3K/AKT/TSC1/TSC2/mTOR signaling pathway, the TSC1/TSC2 protein complex is the key negative regulator of mTOR. Mutations that result in inactive TSC1 or TSC 2 will result in the hyperactivation of $\mathrm{mTOR}$ and this is considered to be the main reason for tumor formation in TSC. Therefore, 
A
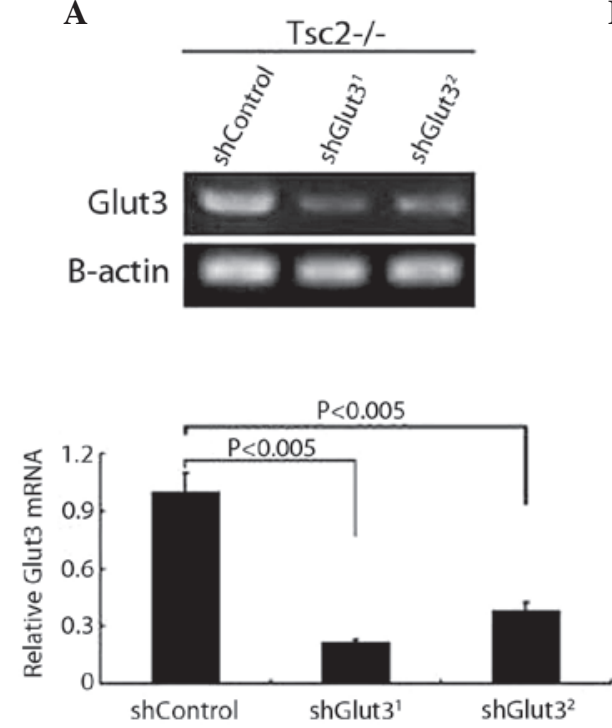

B
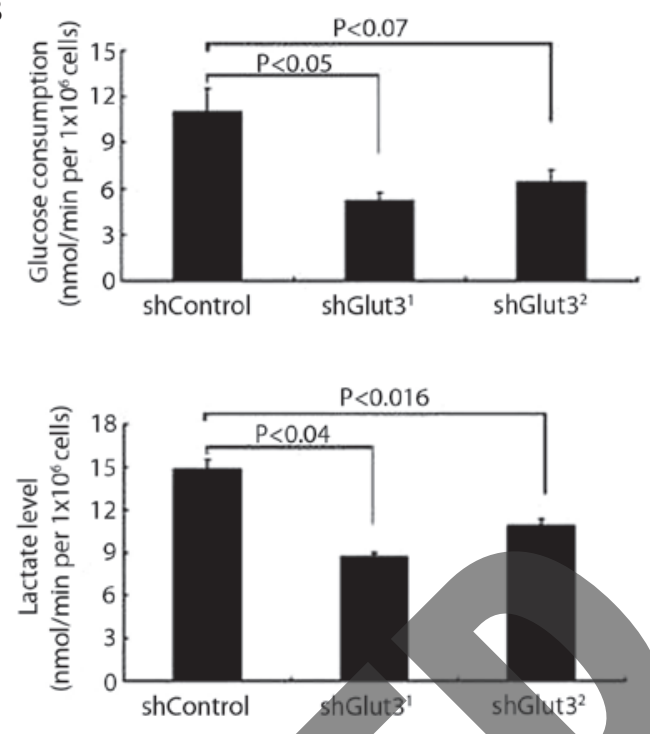

Figure 3. Oncogenic glycolysis is suppressed following depletion of Glut3 levels. (A) Two independent shRNAs that target Glut3 (shGlut $3^{1}$ and shGlut $3^{2}$ ) or a control shRNA (shControl) were stably expressed in Tsc2 ${ }^{-/}$MEFs. The expression of Glut3 was examined by RT-PCR (left panel) and RT-quantitative PCR (right panel). (B) The glucose consumption (left panel) and lactate production (right panel) of $T s c 2^{\%}$ cells expressing shControl, shGlut $3^{1}$, or shGlut $3^{2}$ were measured. Error bars indicate the mean \pm standard deviation of triplicate samples. Glut3, glucose transporter 3; shRNA, short hairpin RNA; Tsc2, tuberous sclerosis complex 2; MEFs, mouse embryonic fibroblasts; RT-PCR, reverse transcription-polymerase chain reaction.

A

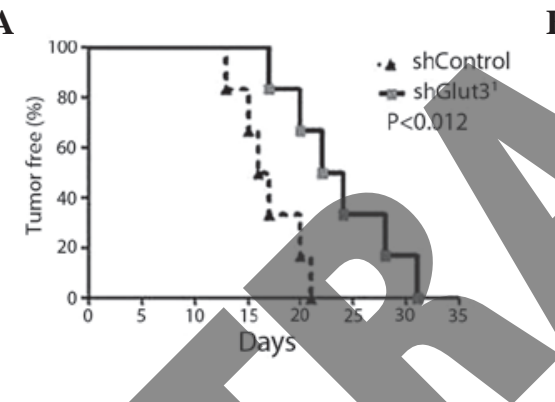

B

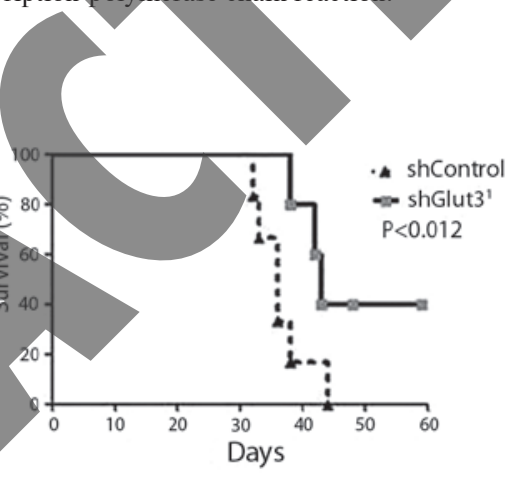

Figure 4. Tumorigenic ability of $T s c 2^{-}$MEFs is suppressed with the depletion of Glut3. Tsc $2^{-}$MEFs infected with two shRNAs (shGlut $3^{1}$ or shControl) expressing lentiviruses were inoculated subcutaneously into nude mice, and assessed for (A) tumor development and (B) survival. Tsc2, tuberous sclerosis complex 2; MEFs, mouse embryonic fibroblasts; Glut3, glucose transporter 3; shRNA, short hairpin RNA.

TSC1- or TSC2-deficient MEFs are appropriate models for the study of mTOR-associated diseases $(9,10)$. Glut-mediated glucose uptake is a rate-limiting step for glycolysis in mammalian cells, and the present study hypothesized that Glut3 is upregulated to compensate for the elevated glucose consumption in mTOR-activated cells. In the present study, p-S6 (an indicator of mTOR activity) was significantly increased in $T s c 2^{-/-}$MEFs compared with $T s c 2^{+/+}$MEFs (Fig. 1A). RT-qPCR analysis also demonstrated that Glut3 expression was increased in $T s c 2^{-/-}$MEFs $(\mathrm{P}<0.001 \mathrm{vs}$. $T s c 2^{--}$cells; Fig. 1A). To further verify the association between the TSC1/TSC 2 complex and Glut3, wild-type human TSC2 was introduced into the Tsc2 $2^{--}$MEFs. Ectopic expression of TSC2 in $T_{s c 2^{--}}$MEFs downregulated the level of p-S6 and reduced the expression of Glut3 (Fig. 1B). Taken together, these results demonstrate that the TSC1/TSC2 complex is a negative regulator of Glut3, the expression of Glut 3 is associated with mTOR activity and that the PI3K/AKT/TSC1/TSC2/mTOR signaling cascade positively regulates Glut3 expression.
mTORC1 promotes Glut3 expression. In mammalian cells, mTOR is combined with various partners to form two complexes, mTORC1 and mTORC2. mTORC1 is activated by the PI3K/AKT pathway (10). To examine which of these complexes is associated with elevated levels of Glut3, the effect of a mTORC1-specific inhibitor (rapamycin) on Glut3 expression was investigated in the present study. p-S6 and Glut 3 expression levels were markedly reduced in $T s c 2^{-/-}$MEFs following treatment with rapamycin (Fig. 2A). In order to further demonstrate the effect of mTORC1 on Glut3, the expression levels of Glut3 following knockdown of mTOR, Raptor (a specific component of mTORC1) or Rictor (a specific component of mTORC2) were assessed. The results demonstrated that Glut 3 expression levels were significantly reduced in cells transfected with mTOR and Raptor siRNAs compared with the control siRNA-transfected cells; whereas the levels in cells treated with Rictor siRNA were not significantly altered (Fig. 2B and C). The results indicate that mTORC1, but not mTORC2, regulates the expression of Glut3. Therefore, Glut 3 may be a potential target for the treatment of tumors that exhibit anomalous mTORC1 signaling. 
Oncogenic glycolysis is suppressed following depletion of Glut3. Aberrantly activated mTOR signaling is considered to be the main reason for tumor formation in $T s c 2^{-/-}$MEFs $(10,11)$. Therefore, $T s c 2^{-/-}$MEFs were used to examine the effect of Glut 3 expression in hyperactive mTOR-mediated glycolysis. Two different shRNAs for Glut3 (shGlut $3^{1}$ and shGlut $3^{2}$ ) were utilized to reduce the Glut3 expression level in $T s c 2^{-1-}$ MEFs. RT-PCR and RT-qPCR analysis demonstrated that the expression levels of Glut3 were significantly reduced in the $T s c 2^{-/-}$MEFs following shGlut $3^{1}$ or shGlut $3^{2}$ treatment, with shGlut $3^{1}$ appearing to be more effective (Fig. 3A). Reduced Glut 3 expression resulted in a marked reduction in glucose consumption and lactate production in $T_{s c} 2^{-/-}$MEFs cells (Fig. 3B). The present study demonstrated that Glut3 is important in mTOR-mediated glycolysis. These results indicate that Glut3 is critical for glycolysis in cells with aberrantly activated mTOR signaling.

Tumorigenic ability of Tsc2 $2^{-/}$MEFs is suppressed following depletion of Glut3. To determine whether Glut3 depletion affects the ability of cells to form tumors in vivo, Tsc $2^{-/}$MEFs expressing shGlut $3^{1}$ or shControl were injected into nude mice subcutaneously. Tsc $2^{-/-}$MEFs expressing shGlut $3^{1}$ exhibited substantially reduced tumorigenic and lethal capacity compared with the control cells (Fig. 4). These results indicate that Glut3 is critical for mTOR-mediated tumor growth in nude mice.

\section{Discussion}

Previously, glucose intermediary metabolism was considered to be a housekeeping function. However, the role of aerobic glycolysis in tumorigenesis, referred to as the 'Warburg effect', is currently of note in the field of cancer research. Warburg hypothesized that irreversible mitochondrial dysfunction is responsible for the metabolic shift to aerobic glycolysis, which results in transformation of the cells $(12,13)$. There has been a considerable resurgence of interest in the role of aerobic glycolysis in cancer. At present, research has shifted from mitochondrial dysfunction to the increased glucose uptake by cancerous cells and the demonstration of the causative effects is the primary objer

mTOR is important in a multitude of cellular processes, including cell growth and protein translation $(6,14)$. A number of previous studies have investigated the nutrient regulation of mTORC1, and have indicated that mTORC1 exerts a vital influence on glucose metabolism $(7,15)$. Several glycolytic enzymes, including pyruvate kinase isoenzyme type M2, hexokinase 2 and lactate dehydrogenase B, have been identified as downstream targets of PI3K/AKT/mTORC1 signaling, which has been reported to promote tumor development by accelerating glycolysis $(16,17)$.

Tuberous sclerosis is an autosomal dominant tumor suppressor gene syndrome that results from inactivation mutations in TSC1 or TSC2 (18). Mice have previously been generated that are null for the $T s c 1$ and $T s c 2$ alleles (19). A previous study in cultured murine cells lacking $T s c 1$ and 2 demonstrated that there is constitutive high-level phosphorylation of S6K and $4 \mathrm{E}-\mathrm{BP} 1$, which is rapidly reversed by rapamycin treatment, identifying mTORC1 as a negative target of Tscl and Tsc2 (19). Another previous study in Drosophila also indicated that
Tscl and Tsc2 acted in pathways associated with mTOR (20), including the PI3K/AKT/mTOR signaling pathway.

In the present study, by analyzing cells with hyperactive mTOR signaling resulting from the deletion of TSC2, it was demonstrated that the TSC1/TSC2/mTORC1 signaling pathway is important in glycolysis and tumorigenesis through the upregulation of Glut3 expression.

The present study provides novel evidence that Glut3 is a downstream effector of mTORC1 that is critical in mTOR-mediated tumorigenesis. Under physiological conditions, Glut 3 is expressed predominantly in the brain tissue (2). The abnormal overexpression of Glut 3 has been detected in numerous types of cancer, including breast, oral, lung, colon and bladder cancer, however, the underlying mechanism remains unclear $(3-5,21)$. Given that mTORC1 signaling is also frequently activated in cancer, one hypothesis is that hyperactivated mTORC1 signaling may be responsible for the elevated Glut 3 in these types of cancer. The results of the present study support this hypothesis (Fig, 2). Therefore, the mTORC1-specific inhibitor rapamycin could be used to treat those types of cancer that express aberrantly activated Glut3.

Cells that exhibit abnormal mTORC1 signaling are more susceptible to glucose withdrawal compared with normal cells (22). Elevated Glut3 due to the activation of mTORC1 may therefore contribute to maintaining transport of glucose at a high rate to ensure tumor cell survival and proliferation. The key role of Glut 3 in glucose metabolism and tumor growth has been characterized in certain human cancer cell lines (23-25). In accordance with these previous studies, the present study shows that the increased expression of Glut 3 is responsible for accelerated glucose consumption, lactate production and tumorigenesis driven by aberrantly activated mTORC1 signaling in MEFs (Figs. 3 and 4). The present study indicated a novel molecular link between mTORC1 activation and enhanced glycolysis and tumorigenesis. Thus, Glut 3 may be a potential therapeutic target for tumors characterized by increased glycolysis resulting from hyperactive mTORC1 signaling.

To the best of our knowledge, the present study is the first to demonstrate that $\mathrm{mTORC} 1$ signaling positively regulates the expression of Glut3, and that the attenuation of Glut3 inhibits oncogenic mTOR-mediated glycolysis and tumorigenesis. However, the underlying mechanism was not examined and future studies should assess whether mTOR regulates Glut3 via $\mathrm{NF \kappa B}, \mathrm{p} 53$ or any other downstream targets of mTOR. In conclusion, the findings of the present study may aid in the explanation of the increased glycolysis observed in tumors with aberrant mTORC1 activity, and provides novel insight into the regulation of Glut3. This may lead to novel diagnostic and therapeutic approaches for those types of cancer that exhibit dysregulated mTORC1 signaling.

\section{Acknowledgements}

The present study was supported by the National Natural Science Foundation of China (grant no. 81101524).

\section{References}

1. Adekola K, Rosen ST, Shanmugam M: Glucose transporters in cancer metabolism. Curr Opin Oncol 24: 650-654, 2012. 
2. Simpson IA, Dwyer D, Malide D, et al: The facilitative glucose transporter GLUT3: 20 years of distinction. Am J Physiol Endocrinol Metab 295: E242-E253, 2008.

3. Ayala FR, Rocha RM, Carvalho KC, et al: GLUT1 and GLUT3 as potential prognostic markers for oral squamous cell carcinoma. Molecules 15: 2374-2387, 2010.

4. Krzeslak A, Wojcik-Krowiranda K, Forma E, et al: Expression of GLUT1 and GLUT3 glucose transporters in endometrial and breast cancers. Pathol Oncol Res 18: 721-728, 2012.

5. Younes M, Brown RW, Stephenson M, et al: Overexpression of Glut1 and Glut3 in stage I nonsmall cell lung carcinoma is associated with poor survival. Cancer 80: 1046-1051, 1997.

6. Honjo S, Ajani JA, Scott AW, et al: Metformin sensitizes chemotherapy by targeting cancer stem cells and the mTOR pathway in esophageal cancer. Int J Oncol 45: 567-574, 2014.

7. Laplante $M$ and Sabatini DM: mTOR signaling in growth control and disease. Cell 149: 274-293, 2012.

8. Moolthiya P, Tohtong R, Keeratichamroen S and Leelawat K: Role of mTOR inhibitor in cholangiocarcinoma cell progression. Oncol Lett 7: 854-860, 2014.

9. Düvel K, Yecies JL, Menon S, et al: Activation of a metabolic gene regulatory network downstream of mTOR complex 1 . Mol Cell 39: 171-183, 2010.

10. Dibble CC, Elis W, Menon S, et al: TBC1D7 is a third subunit of the TSC1-TSC2 complex upstream of mTORC1. Mol Cell 47: 535-546, 2012

11. Lopez-Rivera E, Jayaraman P, Parikh F, et al: Inducible nitric oxide synthase drives mTOR pathway activation and proliferation of human melanoma by reversible nitrosylation of TSC2. Cancer Res 74: 1067-1078, 2014.

12. Warburg O: On the origin of cancer cells. Science 123: 309-314, 1956.

13. Vander Heiden MG, Cantley LC and Thompson CB: Understanding the Warburg effect: the metabolic requirements of cell proliferation. Science 324: 1029-1033, 2009.

14. Cho CH: Frontier of epilepsy research - mTOR signaling pathway. Exp Mol Med 43: 231-274, 2011.
15. Jewell JL and Guan KL: Nutrient signaling to mTOR and cell growth. Trends Biochem Sci 38: 233-242, 2013.

16. Sun Q, Chen X, Ma J, et al: Mammalian target of rapamycin up-regulation of pyruvate kinase isoenzyme type M2 is critical for aerobic glycolysis and tumor growth. Proc Natl Acad Sci USA 108: 4129-4134, 2011.

17. Wolf A, Agnihotri S, Micallef J, et al: Hexokinase 2 is a key mediator of aerobic glycolysis and promotes tumor growth in human glioblastoma multiforme. J Exp Med 208: 313-326, 2011.

18. Krueger DA and Northrup H; International Tuberous Sclerosis Complex Consensus Group: Tuberous sclerosis complex surveillance and management: recommendations of the 2012 International Tuberous Sclerosis Complex Consensus Conference. Pediatr Neurol 49: 255-265, 2013.

19. Kwiatkowski DJ, Zhang H, Bandura JL et al: A mouse model of TSC1 reveals sex-dependent lethality from liver hemangiomas, and up-regulation of p70S6 kinase activity in Tsc1 null cells. Hum Mol Genet 11: 525-534, 2002.

20. Huang $J$ and Manning BD: A complex interplay between Akt, TSC2 and the two mTOR complexes. Biochem Soc Trans 37: 217-222, 2009.

21. Ha TK, Her NG, Lee MG, et al: Caveolin-1 increases aerobic glycolysis in colorectal cancers by stimulating HMGA1-mediated GLUT3 transcription. Cancer Res 72: 4097-4109, 2012.

22. Choo AY, Kim SG, VanderHeiden MG, et al: Glucose addiction of TSC null cells is caused by failed mTORC1-dependent balancing of metabolic demand with supply Mol Cell 38: 487-499, 2010.

23. Masin M, Vazquez J, Rossi S, et al: GLUT3 is induced during epithelial-mesenchymal transition and promotes tumor cell proliferation in non-small cell lung cancer. Cancer Metab 29: 11, 2014.

24. Ha TK, Her NG, Lee MG, et al: Caveolin-1 increases aerobic glyeolysis in colorectal cancers by stimulating HMGA1-mediated GLUT3 transcription. Cancer Res 15: 4097-109, 2012.

25. Jóźwiak P, Krześlak A, Pomorski L, et al: Expression of hypoxia-related glucose transporters GLUT1 and GLUT3 in benign, malignant and non-neoplastic thyroid lesions. Mol Med Rep 6: 601-6, 2012.

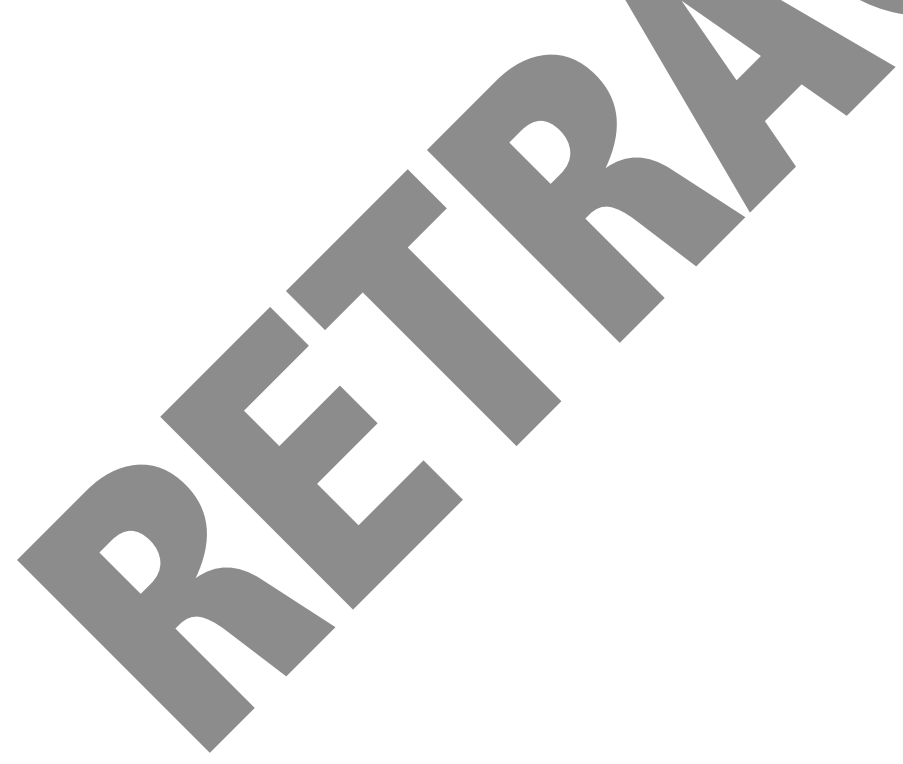

